\title{
'Blastoid' variant of Burkitt lymphoma with additional partial 1q tetrasomy
}

\author{
AHSAN SIDDIQI ${ }^{1}$, SHESHADRI MADHUSUDHANA $^{2 *}$ and ALEXEY GLAZYRIN ${ }^{1^{*}}$ \\ Departments of ${ }^{1}$ Pathology and ${ }^{2}$ Hematology/Oncology, Truman Medical Centers-Hospital Hill, Kansas City, MO 64108, USA
}

Received December 20, 2017; Accepted February 7, 2018

DOI: $10.3892 /$ mco.2018.1585

\begin{abstract}
Burkitt lymphoma is cytogenetically characterized by $\mathrm{t}(8 ; 14)(\mathrm{q} 24 ; \mathrm{q} 32)$ translocation, sometimes accompanied by additional cytogenetic abnormalities. These abnormalities usually result in more aggressive clinical presentation and morphology of the disease. The current report presens a case of Burkitt lymphoma with $\mathrm{t}(8 ; 14)(\mathrm{q} 24 ; \mathrm{q} 32)$ accompanied by partial tetrasomy of chromosome $1(47, \mathrm{XY},+1, \mathrm{i}(1)(\mathrm{q} 10), \mathrm{t}(8 ; 14)$ (q24;q32)[2]/46,XY[18]). The patient was a 59-year-old male who presented with abdominal pain, leukocytosis and tumor lysis syndrome. No lymphadenopathy was noted. Cerebrospinal fluid analysis revealed atypical lymphocytes. A peripheral blood smear revealed tumor cells exhibiting distinct 'blastoid' morphology: Prominent nucleoli, basophilic cytoplasm, occasional cytoplasmic vacuoles. Flow cytometry demonstrated B cells expressing cluster of differentiation (CD)10 and cytoplasmic kappa light chain restriction without surface expression of immunoglobulins and CD20. A bone marrow biopsy revealed hematopoiesis, with a $90 \%$ replacement with atypical lymphocytes. The patient was treated with chemotherapy. Following the first cycle of treatment, the patient developed neutropenic fever, bacteremia and died a few days later. Gain of chromosome $1 \mathrm{q}$ in addition to characteristic for Burkitt lymphoma $\mathrm{t}(8 ; 14)(\mathrm{q} 24 ; \mathrm{q} 32)$ resulted in immature 'blastoid' morphology and the immunophenotype of tumor cells, leukemic presentation without lymph node involvement and a highly aggressive clinical course.
\end{abstract}

\section{Introduction}

Burkitt lymphoma, a B-cell neoplasm, is typically characterized by a $\mathrm{t}(8 ; 14)(\mathrm{q} 24 ; \mathrm{q} 32)$ translocation. There can be other rare

Correspondence to: Dr Alexey Glazyrin, Department of Pathology, Truman Medical Centers-Hospital Hill, 2301 Holmes Street, Kansas City, MO 64108, USA

E-mail: alexey.glazyrin@tmcmed.org

*Contributed equally

Key words: Burkitt lymphoma, partial 1q tetrasomy, double hit, blastoid variant cases commonly identified as 'double hit' lymphoma or 'triple hit' lymphoma. Currently this terminology is most often referred to a combination of translocations involving the c-myc gene located on chromosome 8 combined with $\mathrm{t}(8 ; 14)$ (q24; q32) translocation involving antiapoptotic protein BCL2 or other antiapoptotic proteins (such as BCL6). The updated WHO classification refers to such lymphomas as "high-grade B cell lymphoma with MYC and BCL2 and/or BCL6 translocations' (1). Due to highly aggressive nature of disease classical Burkitt lymphoma is relatively rarely accompanied by other cytogenetic abnormalities. However, when such abnormalities are present it usually entails an even more aggressive behavior/ resistance to existing therapy (2). We will present and discuss a case of a patient with additional cytogenetic abnormality $(47, X Y,+1, \mathrm{i}(1)(\mathrm{q} 10), \mathrm{t}(8 ; 14)(\mathrm{q} 24 ; \mathrm{q} 32)[2] / 46, \mathrm{XY}[18])$ which results in an unusual 'blastoid' morphology of the tumor cells and an extremely aggressive clinical course.

\section{Case report}

We report a case of a 59 year old male who presented in July 2016 to the emergency department because of a one week history of nausea, vomiting with episodic abdominal pain and a decrease in urination with hematuria and dysuria. He has associated weight loss but no recent fevers, chills or a decrease in appetite. He was then admitted for leukocytosis and thrombocytopenia with a possible diagnosis of leukemia, tumor lysis syndrome (elevated potassium and hyperuricemia), pre-renal and post-renal acute kidney injury from dehydration, BPH and renal stone obstruction. A CT of the abdomen and pelvis revealed calculi in the distal right ureter and right ureteral vesicle junction as well as the posterior left aspect of the urinary bladder versus a left ureteral vesicle junction causing bilateral hydroureter and hydronephrosis. Splenomegaly was noted, $22.7 \mathrm{~cm}$ in its greatest diameter. No significant lymphadenopathy was seen. Peripheral blood smear showed numerous large blastoid like cells. He then underwent bilateral ureteral stent placement and a bone marrow biopsy and aspirate procedure.

The peripheral blood smear was performed and it demonstrated a normocytic and normochromic anemia, thrombocytopenia and leukocytosis with numerous large atypical lymphocytes exhibiting a 'blastoid' morphology (prominent nucleoli, basophilic cytoplasm and rare occasional cytoplasmic vacuoles) (Fig. 1A). Flow cytometry demonstrated tumor 

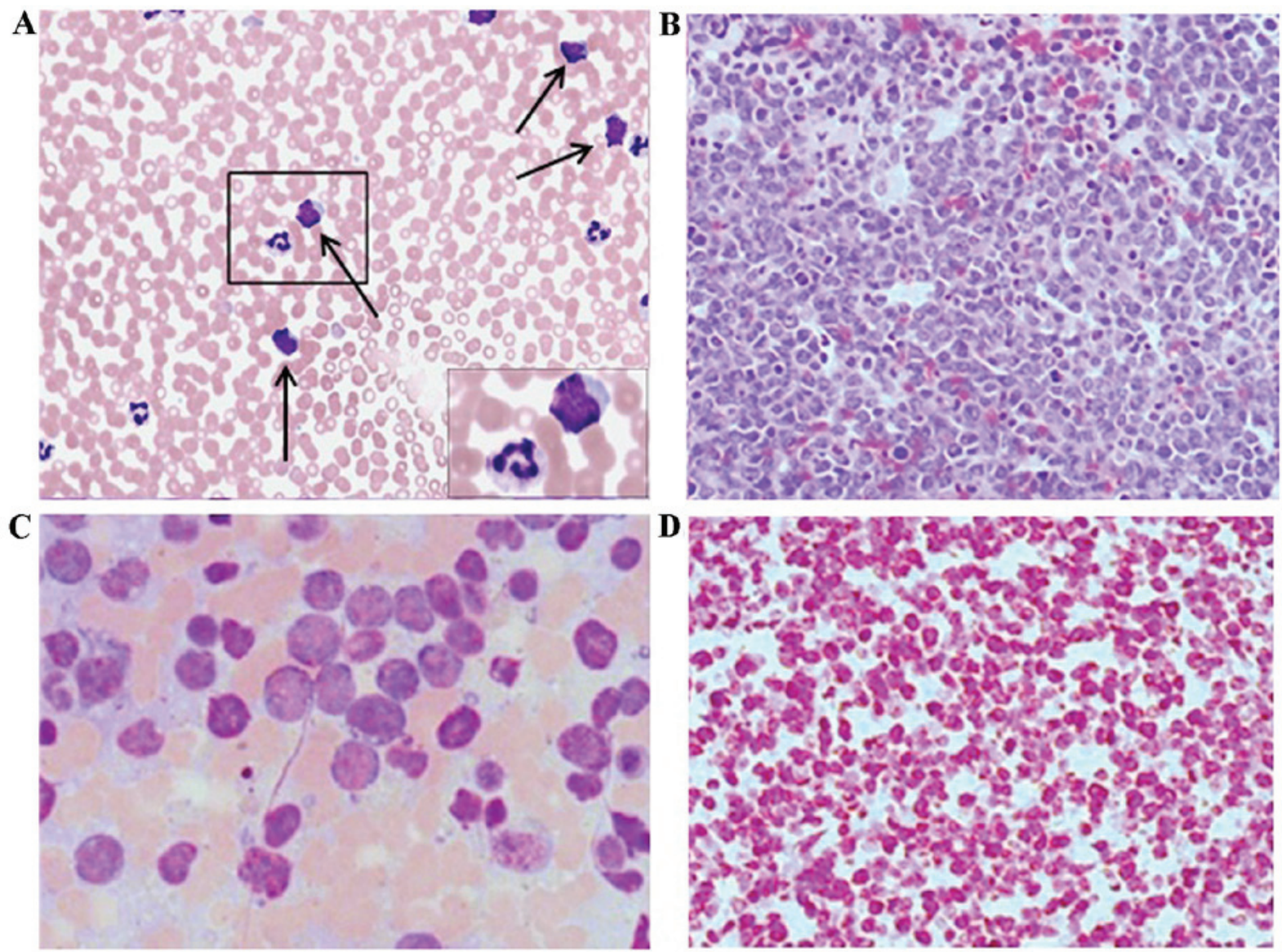

Figure 1. Histologic findings: (A) Peripheral blood with circulating 'blastoid' lymphocytes highlighted by arrows, [hematoxylin and eosin (H\&E) x400]. (B) Bone marrow biopsy. Notice 'starry sky' appearance with total replacement of normal hematopoiesis with atypical lymphocytes, (H\&E, x200). (C) Bone marrow aspirate. Notice proliferation of atypical lymphocytes with occasional cytoplasmic vacuolization (wright-giemsa stain, $x 400$ ). (D) Bone marrow biopsy, Ki67 immunohistochemistry. Notice almost 100\% labeling rate (immunohistochemistry, x200).

cells positive for B cells marker CD79A, CD22, CD10 (dim), CD45. Cytoplasmic light chain staining demonstrated monoclonal expression of cKappa light chain. Cells were negative for surface light chain expression, $\mathrm{TdT}, \mathrm{cCd} 3$ and MPO, CD20, BCL2, BCL6, CD34, CD43. FISH for IgH/ BCL2 $t(14 ; 18)$ and BCL6 (3q27) were negative. This profile is consistent with a CD10-positive kappa light chain restricted B-cell lymphoproliferative disorder with a differential that includes follicular lymphoma, Burkitt lymphoma and large B-cell lymphoma including double hit lymphoma. Concurrent FISH analysis demonstrated $\mathrm{t}(8 ; 14)(\mathrm{q} 24 ; \mathrm{q} 32)$ translocation characteristic for Burkitt lymphoma. The sample was also sent for cytogenetic analysis and it revealed a $47, \mathrm{XY},+1, \mathrm{i}(1)$ $(\mathrm{q} 10), \mathrm{t}(8 ; 14)(\mathrm{q} 24 ; \mathrm{q} 32)[2] / 46, \mathrm{XY}[18]$ with formation of an isochromosome from long arm of chromosome 1, with two normal chromosome 1 homologues, yielding partial tetrasomy 1q. Thereafter a bone marrow biopsy was performed showing 95\% hypercellularity with normal hematopoiesis largely replaced by proliferation of atypical medium to large size lymphocytes (Fig. 1B). Atypical lymphocytes demonstrated irregular, often banded, nuclei with notable nucleoli (Fig. 1C). The cytoplasm is deeply basophilic with occasional lipid vacuoles. Multiple mitotic figures were noted. Ki67 index approached 100\% (Fig. 1D). Diagnosis of Burkitt lymphoma was established.
After initial treatment with hydrea, rasburicase and allopurinol, his labs showed improvement. Imaging was negative for marked lymphadenopathy however there was evidence of splenomegaly. Physical exam findings demonstrated multiple petechiae on the lower extremities.

MRI showed possible meningeal enhancement. An LP was obtained and cytology and flow cytometry showed findings consistent with CNS involvement with lymphoma. He received multiple intrathecal chemotherapies. The patient received chemotherapy as well as intrathecal treatment with methotrexate alternating with cytarabin.

Patient finished cycle 1 of Hyper-CVAD and the course was complicated by sepsis from which he eventually died.

\section{Discussion}

Burkitt lymphoma is an aggressive B cell neoplasm characterized by the translocation and deregulation of the c-MYC gene on chromosome 8 (8q24) along with one of three locations on immunoglobulin (Ig) genes; Ig heavy chain gene on chromosome $14(\sim 80 \%)$ - $\mathrm{t}(8 ; 14)$, kappa light chain gene on chromosome $2(\sim 15 \%)-\mathrm{t}(2 ; 8)$, and the lambda light chain gene on chromosome $22(\sim 5 \%)-\mathrm{t}(8 ; 22)$ (1). Classification of cases with additional copies of cMYC as opposed to translocation remain controversial (3). Present case demonstrated 
classic $\mathrm{t}(8 ; 14)(\mathrm{q} 24 ; \mathrm{q} 32)$ Burkitt lymphoma translocation with additional cytogenetic abnormality (supernumerary isochromosome 1q, i(1)(q10) aberration) resulted in unusual immature 'blastoid' morphology and immature immunophenotype (lack of CD20 and surface immunoglobulin expression) of the tumor cells. Those factors made initial diagnosis in the present case challenging. Peripheral blood so closely mimicked acute leukemia that during initial assessment the peripheral blood smear was demonstrated to medical students, residents and oncology attendings as typical example of acute leukemia, likely ALL, much to the embarrassment of pathologist when Flow and cytogenetic data become available and the very next day the 'text book blasts' turn out to be lymphocytes.

Gain of chromosome 1q has been frequently noted in pediatric malignancies including Wilms tumor, neuroblastoma, Ewing sarcoma and brain tumors such as ependymoma and high grade gliomas and the presence of this anomaly is usually associated with disease recurrence and poor prognosis. However the candidate genes on chromosome 1q that could be involved in tumorigenesis remain unidentified (4). An extra copy 1q was demonstrated before in 10 patients with hematologic malignancies out of 3786 studied: 5 with myelodysplastic syndrome, 3 with acute myeloid leukemia, and a single patient with acute lymphoblastic leukemia and myeloproliferative syndrome (5).

To our knowledge 12 other cases of Burkitt lymphoma with supernumery idic (1)(p12) or i(1)(q10) were described in literature before with $>80 \%$ cases presented with immature B-cell immunophenotype and $>60 \%$ of patients relapsed or died (6). Our case demonstrated a very similar pattern with 'blastoid' immature phenotype and immunophenotype and highly aggressive course resulting in rapid death. This case combined with literature data allows us to view those cases of Burkitt lymphoma with $t(8 ; 14)(q 24 ; q 32)$ combined with polysomy of 1q as possible separate category of Burkitt lymphoma similar to 'double hit' and 'triple hit' lymphomas. Such cases are characterized by immature 'blastoid' tumor cells morphology and immunophenotype, leukemic presentation without lymph nodes involvement as well as highly aggressive clinical behavior.

\section{Acknowledgements}

The authors would like to thank the hematology department of Truman Medical Centers-Hospital Hill (KansasCity, MO, USA) for their cooperation.

\section{Funding}

Not applicable.

\section{Availability of data and materials}

All data analyzed during this study are included in the article.

\section{Authors' contributions}

All authors contributed equally to this manuscript. This is further indicated by the asterisk marks with author names in the title of the paper.

\section{Ethics approval and consent to participate}

Not applicable.

\section{Consent for publication}

In accordance with regulations in effect with the University of Missouri-Kansas City all the identifying information was removed, publication of data does not compromise anybody anonymity or confidentiality or breach local data protection laws. Any minimal risk of breaking of confidentiality if at all present is outweighed by public interest consideration. The patient is long time deceased and therefore at this time it is impossible to obtain permission from him or trace his significant others. All the identifying material has been thoroughly removed from the manuscript therefore no reasonable individual would object to publication.

\section{Competing Interests}

The authors declare that there are no competing interests regarding the publication of this paper.

\section{References}

1. Swerdlow SH, Campo E, Pileri SA, Harris NL, Stein H, Siebert R Advani R, Ghielmini M, Salles GA, Zelenetz AD, et al: The 2016 revision of the World Health Organization classification of lymphoid neoplasms. Blood 127: 2375-2390, 2016

2. Onciu M, Schlette E, Zhou Y, Raimondi SC, Giles FJ, Kantarjian HM, Medeiros LJ, Ribeiro RC, Pui CH and Sandlund JT: Secondary chromosomal abnormalities predict outcome in pediatric and adult high-stage Burkitt lymphoma. Cancer 107: 1084-1092, 2006.

3. Chang QA, Qasem A, Madhusudhana S and Glazyrin A: The $\mathrm{t}(14 ; 18)(\mathrm{q} 32 ; \mathrm{q} 21)$ with extra MYC signal - is it a gray zone lymphoma? Int J Clin Exp Pathol 8: 9602-9608, 2015.

4. Puri L and Saba J: Getting a clue from 1q: Gain of chromosome 1q in cancer. J Cancer Biol Res 2: 1053, 2014.

5. Djordjević V, Dencić-Fekete M, Jovanović J, Drakulić D, Stevanović M, Janković G and Gotić M: Pattern of trisomy 1q in hematological malignancies: a single institution experience. Cancer Genet Cytogenet, Oct 186: 12-18, 2008.

6. Roug AS, Wendtland P, Bendix K and Kjeldsen E: Supernumerary isochromosome 1, idic(1)(p12), leading to tetrasomy 1q in Burkitt lymphoma. Cytogenet Genome Res 142: 7-13, 2014.

This work is licensed under a Creative Commons Attribution-NonCommercial-NoDerivatives 4.0 International (CC BY-NC-ND 4.0) License. 